\title{
Lidil
}

Revue de linguistique et de didactique des langues

\section{Grammaticalisation et acquisition du prédicat complexe faire + Vinf}

\section{Yanka Bezinska}

\section{OpenEdition}

\section{Journals}

Édition électronique

URL : http://journals.openedition.org/lidil/2698

DOI : $10.4000 /$ lidil.2698

ISSN : 1960-6052

\section{Éditeur}

UGA Éditions/Université Grenoble Alpes

\section{Édition imprimée}

Date de publication : 1 juin 2008

Pagination : 149-174

ISBN : $978-2-84310-124-3$

ISSN : 1146-6480

\section{Référence électronique}

Yanka Bezinska, "Grammaticalisation et acquisition du prédicat complexe faire + Vinf », Lidil [En ligne], 37 | 2008, mis en ligne le 01 septembre 2009, consulté le 22 avril 2019. URL : http:// journals.openedition.org/lidil/2698; DOI : 10.4000/lidil.2698 


\title{
GRAMMATICALISATION ET ACQUISITION DU PRÉDICAT COMPLEXE FAIRE + VINF
}

\author{
Yanka BEZINSKA*
}

\section{RESUME}

Le présent article est constitué de deux parties. Dans la première, nous nous penchons sur l'étude du processus de grammaticalisation du prédicat complexe faire + Vinf. Nous y distinguons trois principales étapes: 1) emploi généralisé en latin de constructions bi-clausales du type $\mathbf{V} \mathbf{1}_{\text {de commande, de persuasion }}+$ complémenteur + $\mathbf{V} \mathbf{2}_{\text {subjonctif }} ; 2$ ) coexistence des formes ancienne (deux prédicats) et nouvelle (prédicat complexe); 3) la nouvelle forme exclut l'ancienne. Nous mentionnons les critères d'identification de faire + Vinf en tant que prédicat complexe et nous illustrons par la suite son statut au sein des autres mécanismes causatifs dans les langues; nous évoquons également quelques éléments de comparaison avec les constructions causatives en bulgare.

Dans la deuxième partie de l'article, nous présentons une expérimentation menée sur l'acquisition du prédicat complexe faire + Vinf chez des enfants unilingues francophones entre 4 et 6 ans. A travers les résultats obtenus, nous essayons d'établir un parallélisme entre les étapes de grammaticalisation et d'acquisition de cette construction.

\section{ABSTRACT}

This paper comprises two parts. In the first, we examine the process of grammaticalisation of faire + Vinf complex predicate. We distinguish three stages: 1) the generalized use in Latin of biclausal constructions $\mathbf{V} \boldsymbol{I}_{\text {command, persuasion }}+$ complementizer $+V 2_{\text {subjonctive ; }}$ ) the old form (two predicates) and the new form (complex predicate) coexist; $3 /$ the new form entirely replaces the former. We then specify the criteria of identification of faire + Vinf complex predicate and illustrate its status within other causative mechanisms; we also mention some comparative elements of the causative construction in Bulgarian.

In the second part of this paper, we present an experiment conducted on the acquisition of faire + Vinf by 4 to 6 year-old French children. The results lead us to postulate a parallel between the stages of grammaticalisation and the acquisition of this construction.

* Université Stendhal-Grenoble, Lidilem. 
De nombreux travaux en typologie syntaxique se penchent sur la problématique de la fusion des prédicats, ou sur ce qu'on appelle encore formation des prédicats complexes. Notre article s'inscrit dans ce type de questionnements en présentant une étude sur la grammaticalisation du prédicat complexe faire + Vinf et son acquisition par des enfants unilingues entre 4 et 6 ans.

Dans un premier temps, nous donnerons une brève définition des prédicats complexes dans les langues. Nous tenterons par la suite d'esquisser les étapes de grammaticalisation de la construction factitive faire + Vinf, ainsi que de dégager les critères de son identification en tant que PC. Nous justifierons également son statut au sein des mécanismes causatifs et nous évoquerons quelques éléments de comparaison avec les constructions causatives en bulgare. Dans la dernière section, nous traiterons de l'acquisition de faire + Vinf par des enfants unilingues francophones entre 4 et 6 ans. Nous essaierons d'établir un parallélisme entre les étapes de grammaticalisation et d'acquisition de la construction.

\section{La grammaticalisation de faire + Vinf}

Selon Abeillé et Godard (2003: 1), les prédicats complexes représentent «[...] une suite de verbes ou prédicats [qui] fonctionne comme un seul verbe vis-à-vis de certaines propriétés syntaxiques ou sémantiques. Les phrases comportant un prédicat complexe ont ainsi des propriétés de monoclausalité». Comme 1'indiquent les auteurs, les prédicats complexes sont reconnaissables à deux caractéristiques corrélées, à savoir les clitiques (objet et datif) sont portés par le verbe tête et le complément est non-fini (infinitif, gérondif, participe ou prédicat non-verbal), ou plus précisément non fléchi.

Les termes de clause union et de monoclausalité (ou encore, formation de prédicats complexes) évoquent un type d'évolution particulièrement fréquente dans les langues liées au changement linguistique. Ce dernier représente un phénomène universel dû à un ensemble de facteurs : facteurs d'ordre 
cognitif ${ }^{1}$, social ou pragmatique, facteurs internes au système de la langue (Combettes et al. 2003 : 234).

Toutefois, notre intérêt porte sur le changement d'ordre syntaxique; il existe deux approches pour traiter ce phénomène: une approche formelle et une approche fonctionnelle (Peyraube, 2002: 47-49). La première considère que c'est l'acquisition par l'enfant de sa langue maternelle qui est à la base du changement grammatical, celui-ci étant non progressif et autonome de toute considération fonctionnelle. La seconde relie le changement syntaxique à des facteurs discursifs, historiques, développementaux. L'auteur mentionne également les trois mécanismes fondamentaux de ce phénomène observable dans les langues: l'analogie (extension), la réanalyse (grammaticalisation) et l'emprunt externe (Peyraube, 2002: 49-54).

De tous les mécanismes du changement syntaxique évoqués ci-dessus, la grammaticalisation est le seul à pouvoir expliquer le processus de la formation du prédicat complexe (PC) faire + Vinf.

Le terme de grammaticalisation a été défini pour la première fois par A. Meillet (1912-1982: 131) comme «le passage de la composante lexicale à la composante grammaticale». Kurylowicz (1965: 52) élargit cette notion en parlant d'«un processus, qui soit change des lexèmes pleins en mots grammaticaux, soit rend des éléments grammaticaux encore plus grammaticaux». Dans la littérature, on retrouve également le terme de «grammaire émergente» (emergent grammar) désignant «un mouvement vers une structure» (movement toward a pattern) (Hopper, $1987: 148$ ).

Il existe quatre paramètres de nature différente (sémantique, morphosyntaxique, morphophonologique, phonétique), qui sont révélateurs du processus de grammaticalisation (Lamiroy, 1999: 35-37). Pourtant, l'évolution de faire + Vinf n'illustre que deux de ces quatre paramètres: la désémantisation et la décatégorisation syntaxique de faire. Le premier processus concerne l'entrée lexicale qui «se vide progressive-

1. Dans ce cas-là, l'auteur évoque le problème de l'apprentissage de la langue maternelle par l'enfant. 
ment de son sens plein pour acquérir en revanche un sens fonctionnel, grammatical» (Lamiroy, 1999: 35). La décatégorisation syntaxique consiste dans le fait que: «... le verbe tend de moins en moins à sélectionner les syntagmes nominaux pour s'associer de plus en plus à des types de verbe non finis ou non tensés, tel l'infinitif» (Heine, 1993: 75). Finalement, une montée des clitiques se produit aboutissant à une réanalyse des deux verbes comme un seul syntagme verbal. Les travaux sur la grammaticalisation des unités linguistiques font état de trois phases, ce qui est également valable pour la construction faire + Vinf:

Étape 1: 1'emploi généralisé en latin de constructions biclausales V1 (de commande, de persuasion) + complémenteur + V2 subjonctif (inducere aliquem ut mentiatur).

Étape 2: les formes ancienne (deux prédicats) et nouvelle (prédicat complexe) coexistent en ancien et en moyen français (entre les $\mathrm{XI}^{\mathrm{e}}$ et $\mathrm{XV}^{\mathrm{e}}$ siècles).

Étape 3 : la nouvelle forme exclut l'ancienne.

La première étape illustre une période antérieure au changement observable en latin classique, une langue de basse orientation causative (poor causative-oriented language) Simone \& Cerbasi, 2001: 450). Le premier verbe de la construction $V I+u t+V 2$ subjonctif n'est pas spécifiquement causatif; il est teinté de différentes valeurs modales (coercition, persuasion, ordre). Pour relier les agents de deux actions différentes et pour exprimer les rapports spécifiques établis entre eux (hiérarchie, degré d'agentivité, degré de contrôle), le latin fait appel à des constructions moins grammaticalisées du type : iubere $+u t+V$ subjonctif, facere $+u t+V$ subjonctif, inducere $+u t+V$ subjonctif:

(1) Tu etsi non potuisti ullo modo facere ut mihi illam epistulam non mitteres, tamen mallem non esse missam.

[Ah! Certes tu ne pouvais pas faire autrement que de me l'envoyer, mais $\mathrm{j}$ 'aurais été certainement plus heureux à ce qu'elle ne m'eût pas été envoyée.] (Cicéron, Epistulae ad Atticum, T. 2, livre XI, epis. 21, paragr. 1, p. 384) 
L'évolution de ces constructions à deux verbes distincts se poursuit, marquée par la suppression du complémenteur $u t$ et 1'apparition de l'infinitif à la place du subjonctif:

(2) Ut ille fidicinam fecit nescire esse emptam tibi.

[Comme il a laissé ignorer à la joueuse de lyre qu'on l'achetait pour toi.] (Plaute, Comédies (Epidicus), T. 3, acte III, 30, p. 144).

Par ailleurs, on pourrait déjà relever en latin des exemples d'insertion d'un syntagme nominal (SN) entre le verbe faire et l'infinitif:

(3) Qui nati coram me cernere letum fecisti et patrios foedasti funere uoltus.

[... qui m'as fait voir de mes yeux le trépas de mon enfant, qui as souillé de mort le visage d'un père!] (Virgile, Enéide, T. 1, livres I-IV, p. 59).

Quæ tremulum faciunt membris existere motum.

(4) $[\ldots$ qui provoquent le tremblement de tous ses membres.] (Lucrèce Carus, De rerum natura, T. 1, livres I-III, p. 97).

En ancien français, le verbe causatif faire peut être suivi de l'infinitif d'un verbe transitif ou intransitif ${ }^{2}$ :

Morz est mis niés, ki tant me fist cunquere.

[Il est mort, mon neveu, qui me conquit tant de terres.]

(Roland, v. 2920 , p. 287$)^{3}$.

En seintes flurs, il les facet gesir.

[... et que parmi les saintes fleurs il les fasse reposer.] (ibid, v. 1856, p. 207) .

2. C'est également le cas en français contemporain (ex. Il fait rire la fille. - faire + verbe intransitif; La maman fait manger du gâteau au bébé. - faire + verbe transitif).

3. Dans l'exemple (5) ci-dessus le verbe causatif faire est suivi de l'infinitif d'un verbe transitif.

4. Dans l'exemple (6) ci-dessus le verbe causatif faire est suivi de l'infinitif d'un verbe intransitif. 
Cependant, en ancien et en moyen français, l'insertion d'un syntagme nominal (SN) entre faire et l'infinitif est tout à fait ordinaire lorsque ce dernier n'est pas suivi d'un complément d'objet direct; on en trouve des exemples significatifs en vers, en prose, dans le théâtre :

Si l'orrat Carles, ferat l'ost returner.

[Charles l'entendra, il fera retourner l'armée.] (Roland, v. 1060, p.147)

Ge ferai le baron venir... (Béroul, Tristan, v. 3085, $\mathrm{XIII}^{\mathrm{e}}$ siècle $)^{5}$.

Et puis fisent toutes manières de gens, petits et grans, partir (Froissart, Chron., I, 313, t. IV, p. 63, I. 29-30, $\mathrm{XIV}^{\mathrm{e}}$ siècle).

Pour faire tout le monde rire (théâtre $d u \mathrm{XV}^{\mathrm{e}}$ et $\mathrm{du}$ $\mathrm{XVI}^{\mathrm{e}}$ siècle).

La construction faire + Vinf ne garde pas le même statut au cours des siècles. Peu à peu le SN inséré entre faire et l'infinitif se déplace à droite de l'infinitif. Le verbe faire, de sens très général, se désémantise et se décatégorise progressivement; autrement dit, il commence à admettre la montée du clitique, ce qui est un indice révélateur "d'une restructuration, c'est-à-dire d'une réanalyse des deux verbes comme un seul syntagme verbal, et donc d'une grammaticalisation" (Lamiroy, 1999: 44). Finalement, la soudure syntaxique de faire et du verbe à l'infinitif, s'établit comme norme au XVII ${ }^{\mathrm{e}}$ siècle. (Nilsson-Ehle, 1948).

La deuxième étape de la grammaticalisation de faire $+\operatorname{Vinf}$ se caractérise donc par la fluctuation entre l'ancienne forme (à deux prédicats) et la nouvelle (une seule unité prédicative, $\mathrm{PC})$ :

M'annonçant que des dieux la juste bienveillance aura de mon costé fait tourner la balance (J. de Schelandre, Tyr et Sidon, ATF, T. 8 , v. 28 , p. 47,1628$)^{6}$.

5. Les exemples de (8) à (10) sont empruntés à Gougenheim (1929: 357-358).

6. Les exemples de (11) à (14) sont empruntés à Gougenheim (1929: $331 \& 358$ ). 
Qui [un serpent] siffle et fait grincer la dent envenimée (Desmarets de Saint-Sorlin, Clovis, 1657-1673, A. Haase, Ergaenzende Bemerkungen zur Synt. Des XVII. Jh's, Zs.f.fz. Spr., XI, p. 218).

Qui facit les coquins mandier? (Rabelais, Pantagruel, III, 14. XVI ${ }^{\mathrm{e}}$ siècle).

Ce sera vous qui de nos villes ferez la beauté refleurir, vous qui de nos haines civiles ferez $\underline{\text { la racine mourir }}$ (Malherbe, T. 1, v. 92 et 94, p. 49; Regnier, Lex., s. v; faire, 3).

En dépit de ces fluctuations, la nouvelle forme de la construction factitive faire + Vinf s'impose; son statut de prédicat complexe (PC) est indéniable (Gaatone, 1976; Alsina, 1996; Abeillé \& Godard, 2003, Creissels, 2006).

Par ailleurs, il est intéressant que certains auteurs contemporains remettent le SN au milieu du PC, et ce pour des raisons stylistiques ${ }^{7}$ :

Les mots de passion qui faisaient l'amant frôler son visage au papier satiné (A. Daudet, Sapho, 1884) ${ }^{8}$.

Ce qui faisait Armand répondre à ceux qui lui demandaient... (A. Gide, Les Faux Monnayeurs, 1925).

La grammaticalisation de la construction factitive faire + Vinf en français est un processus unidirectionnel allant des structures analytiques (à deux prédicats, en latin) aux formes synthétiques (prédicat complexe, en français contemporain); sur le plan sémantique, il y aurait également une tendance à la lexicalisation de la construction (ex.: faire voir = montrer, faire mourir $=$ tuer, faire connaitre $=$ annoncer $)$.

7. Dans une correspondance avec A. Gide, Nilsson-Ehle lui demande «si, en écrivant des phrases du genre [ Ce besoin de noblesse qui, passé vingt-six ans, fait encore Nietzsche préférer Schiller à Goethe $»]$, il a eu l'intention de ranimer la vieille manière de s'exprimer, ou si ...] ces phrases lui sont venues au bout de la plume, naturellement (et avec le seul souci de la précision de la pensée). » (Nilsson-Ehle, 1948: 101). A ce questionnement, $A$. Gide répond de la manière suivante : «N'en doutez pas : c'est tout naturellement que je me suis exprimé ainsi - sans même trop me rendre compte ... ]) (idem).

8. Les exemples de (15) à (16) sont empruntés à Gougenheim (1929: $358)$. 


\section{Les critères d'identification de faire + Vinf en tant que prédicat complexe}

La question qui se pose après avoir esquissé la diachronie des PC est celle de leur reconnaissance parmi les autres constructions complexes. Creissels (2006) propose un test fiable basé sur le comportement des indices pronominaux (les clitiques objet et datif). Si les clitiques s'attachent aux deux verbes composants, alors chacun d'eux est autonome. Par contre, si les clitiques s'attachent au verbe auxiliaire, alors on est en présence de fusion de prédicats (formation de PC). Pour ce qui est du français, l'auteur donne à titre d'exemple les deux constructions de laisser + Vinf et de voir + Vinf:

(17) J'ai laissé les enfants manger le gâteau'.

(17a) Je les ai laissé le manger.

(18) J'ai laissé manger le gâteau aux enfants.

(18a) Je le leur ai laissé manger.

(19) J'ai vu Jean réparer la moto.

(19a) Je l'ai vu la réparer.

(19b) Je la lui ai vu réparer.

Il existe aujourd'hui un large consensus pour traiter faire + Vinf comme prédicat complexe ${ }^{10}$. Ainsi Gaatone (1976: 166-169) affirme que « [1] phrase factitive pourrait être considérée comme une phrase simple à verbe complexe plutôt que comme une phrase complexe comportant deux verbes ». Il propose une série de tests afin de prouver la grammaticalisation de la construction:

a) insertion des indices pronominaux (les clitiques objet + datif) entre le sujet et faire + Vinf (ex.: Elle fait manger de la soupe à l'enfant. $\rightarrow$ Elle la lui fait manger. * Elle lui fait la manger).

9. Les exemples de (17) à (19) sont empruntés à Creissels (2006: 290-291). En (17a) les clitiques s'affixent aux deux verbes, alors il ne $s$ 'agit pas de PC. En (18a) les clitiques s'attachent au verbe auxiliaire, donc la périphrase laisser manger est un PC. Il en est de même en (19a) (voir + inf n'est pas un PC) et en (19b) (voir + inf est un PC).

10. Gaatone (1976); Alsina (1996); Abeillé \& Godard (2003). 
b) impossibilité de supprimer l'infinitif (ex.: Elle a fait manger l'enfant. $\rightarrow{ }^{*}$ Elle l'a fait $+\underline{\text { Q. }}$. .

c) place du pronom conjoint en inversion (ex.: Fait-elle manger le bébé? *Fait manger-elle le bébé?).

d) forme négative (ex.: Elle ne fait pas manger le bébé. *Elle ne fait manger pas le bébé.).

e) forme impérative (ex.: Fais-les écrire! *Fais écrireles! (pas de liaison); Fais-le écrire! *Fais écrire-le! (pas d'élision).

Cela rapproche la construction faire + Vinf des formes verbales, composées d'un auxiliaire (avoir/être) et d'un participe passé. Le verbe faire fonctionne comme auxiliaire causatif véhiculant les informations de temps-aspect-mode (TAM).

Une grande partie des travaux sur la construction factitive s'inscrivent dans le cadre de la grammaire de dépendance, plus précisément, de la syntaxe structurale de Tesnière (1969). Le factitif est conçu comme une opération sur la valence verbale: l'introduction du verbe faire augmente d'un nouvel actant (valence $n+1$ ) la valence du verbe à l'infinitif. Cet argument supplémentaire qui s'ajoute aux participants déjà présents dans le schéma argumental de base est reconnaissable comme causateur et assume le rôle syntaxique du sujet. Le sujet initial est destitué; il peut occuper différentes places syntaxiques (objet, datif, oblique) en fonction de la valence du verbe initial ${ }^{11}$.

Sur le plan sémantique, la construction faire + Vinf est conçue comme vẻhiculant un enchâssement sous faire d'une relation sémantique, où le nouvel actant (agent, causateur, causer) provoque ou déclenche la réalisation du procès exprimé par le verbe à l'infinitif (Novakova, 2002). En

11. En fonction du verbe: intransitif, transitif, ditransitif, le sujet destitué prend la première place disponible dans la hiérarchie OD $>$ OI $>0 O$ (Comrie, 1976). Il s'ensuit qu'après la dérivation causative, les verbes avalents deviennent monovalents (à 1 actant) (ex. Il pleut. $\rightarrow L a$ condensation de la vapeur d'eau fait pleuvoir, in Tesnière, 1969: 265), les verbes monovalents deviennent bivalents (à 2 actants) (ex. Bernard tombe. $\rightarrow$ Alfred fait tomber Bernard, idem), les verbes bivalents deviennent trivalents (à 3 actants) (ex. Bernard frappe Alfred. $\rightarrow$ Charles fait frapper Alfred par Bernard, p. 266). 
revanche, l'actant destitué peut assumer différents rôles sémantiques, à savoir: patient, victime, deuxième agent, bénéficiaire. Cette diversité est réunie sous le terme plus neutre de causataire (causee), d'où finalement le binôme causateur-causataire (idem).

\section{Le statut de faire + Vinf au sein des mécanismes causatifs}

Les langues font usage de procédés très variés pour exprimer la causativité. Ainsi Dixon (2000) range les mécanismes causatifs du plus compact (synthétique) au moins compact (analytique). La figure 1 ci-dessous illustre l'échelle de leur compacité (scale of compactness):

Le plus compact
Lexical

Morphologique

Prédicat complexe (PC)

Constructions périphrastiques

Figure 1 -Échelle de compacité (scale of compactness) (Dixon, 2000).

Le mécanisme lexical correspond à un seul verbe de sens causatif (ex.: tuer, montrer). Sur le deuxième palier (procédé morphologique) se rangent quelques verbes causatifs suffixés (ex.: raréfier, moraliser). La construction faire + Vinf se trouve sur le troisième palier de l'échelle. De par son apparence, ce procédé est analytique, mais le degré de soudure entre les éléments composants reste très fort. Si l'on compare les PC dans les langues romanes, on arrive à la conclusion que seules les périphrases à base de faire (français) et de fare (italien) représentent des $\mathrm{PC}$ à proprement parler ${ }^{12}$. La construction à base de hacer (espagnol) peut être fort grammaticalisée (un PC), ou bien, moins grammaticalisée permettant l'insertion d'un SN entre hacer et l'infinitif ${ }^{13}$. Enfin, le roumain est la seule langue romane à ne pas faire usage de PC pour expri-

12. Par exemple dans (20): Le professeur a fait écrire un test aux élèves et (21): Il professore ha fatto scrivere un test agli allievi, empruntés à Iliescu (1996: 9-10).

13. Par exemple dans (22): El profesor ha hecho escribir un test a los alumnos (formation de $\mathrm{PC}$ ) et (23): Este comportamiento de las prepo 
mer la causativité ${ }^{14}$. Les causatifs roumains représentent des complétives au subjonctif (face + subjonctif, pune + subjonc$\left.t_{i f}\right)^{15}$. Le phénomène est à mettre en relation avec l'élimination progressive de l'infinitif en roumain ${ }^{16}$. Ce même trait, propre à l'aire balkanique, est observable également en bulgare. Les constructions causatives du roumain et du bulgare correspondent au quatrième palier, le moins compact, de l'échelle de Dixon.

En bulgare, les trois mécanismes (lexical, morphologique, périphrases ne formant pas de PC) sont présents. Par exemple, le procédé lexical est illustré par des verbes comme vzrivjavam se (exploser)-vzrivjavam (faire exploser); le mécanisme morphologique est représenté par l'apophonie (pija - boire poja-faire boire), d'usage plutôt archaïque, ainsi que par la préfixation (plača - pleurer razplakvam - faire pleurer). Enfin, le bulgare utilise des constructions périphrastiques moins grammaticalisées du type $\boldsymbol{V}_{\text {sens causatif }}+\boldsymbol{d a} \boldsymbol{a}_{\text {conjonction }}$

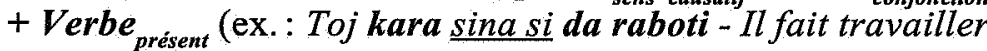
son fils. $=$ Il fait en sorte que son fils travaille. $=I l$ incite son fils à travailler/à ce qu'il travaille).

D'après Gawełko (2006), il y aurait un rapport entre le choix du mécanisme causatif et le caractère typologique de la langue où ces causatifs apparaissent. Ainsi, le français est, premièrement, une langue à forte tendance analytique, ce qui expliquerait la grande vitalité du PC faire + Vinf. Le bulgare a développé un système de préfixes extrêmement riche, d'où l'importance des causatifs morphologiques. Cette langue a également recours à des constructions périphrastiques moins grammaticalisées ( $c f$. supra), pour exprimer la causativité. Pourtant, ces structures ne forment pas de PC (comme le PC faire + Vinf en français), ce qui pourrait être expliqué par la

siciones... hizo a los linguistas italianos hablar de la existencia de tal caso en su lengua (pas de formation de PC), empruntés à Iliescu (1996: 10-11).

14. Cf. à ce sujet Iliescu (1996); Abeillé \& Godard (2003); Gawełko (2006).

15. Par exemple dans (24): Profesorul i-a făcut pe elevi să scrie un test et (25): Profesorul $i$-a pus pe elevi să scrie un test, empruntés à Iliescu (1996: 10). 
disparition de l'infinitif synthétique (entre les $\mathrm{XII}^{\mathrm{e}}$ et $\mathrm{XV}^{\mathrm{e}}$ siècles) et son remplacement par la construction :

$$
d a_{\text {conjonction }}+V_{\text {présent }}{ }^{17} \text {. }
$$

\section{L'acquisition de faire + Vinf}

Comme indiqué dans l'introduction, la dernière section de notre article sera consacrée à l'expérimentation menée sur 1 'acquisition de faire $+V$ inf chez des enfants unilingues francophones entre 4 et 6 ans. Nous tenterons de voir dans quelle mesure les étapes de l'évolution de cette construction dans le langage enfantin pourraient reproduire celles de sa grammaticalisation.

\section{Méthodologie de l'expérimentation}

L'expérimentation menée sur l'acquisition du prédicat complexe faire + Vinf inclut 18 enfants unilingues francophones; ceux-ci sont répartis en deux tranches d'âge : 9 enfants (5 garçons +4 filles) âgés entre 4 et 5 ans et 9 enfants ( 5 garçons +4 filles) âgés entre 5 et 6 ans. Tous les participants sont enregistrés une seule fois en milieu scolaire (à la maternelle). Il s'agit d'entretiens individuels d'une durée approximative de 30 minutes.

Le choix des verbes cibles de notre recherche n'est pas arbitraire. Il est conditionné par deux facteurs principaux: 1/les propriétés syntaxiques et sémantiques des verbes (Hypothèse inaccusative de Perlmutter, 1978; de Levin \& Rappaport, 1995); 2/la fréquence avec laquelle ceux-ci apparaissent dans les constructions factitives (calculée dans le corpus FrANTEXT).

Nous avons donc choisi huit verbes de nature sémantique et syntaxique variée:

16. Voir à ce sujet Gawełko (2006).

17. Voir à ce sujet Novakova (2006). 
a) intransitifs-rire et pleurer (inergatifs agentifs ${ }^{18}$ ); danser (inergatif de mouvement ${ }^{19}$ ) et tomber (inaccusatif de changement de position ${ }^{20}$ ). Dans ce cas-là, notre but est de voir si les enfants unilingues francophones auront tendance à supprimer le verbe factitif faire (ex.: saute ça, vais boire mon bébé, je danse le p'tit chat, cf. Sarkar, 2002: 191).

b) transitifs-boire et manger. Ceux-ci peuvent apparaitre dans des structures intransitives (Le bébé boit/mange.) et transitives (Le bébé boit du lait; L'ours mange du miel.). Après la dérivation causative, ces verbes donnent naissance à des constructions factitives plus ou moins complexes (manger $\rightarrow X$ fait manger $Y$, ou bien, $X$ fait manger $Y \grave{a} Z$ ). Notre expérimentation vise à explorer la capacité des jeunes locuteurs à produire les deux types de constructions factitives: 1/La maman fait boire/manger le bébé; 2/La maman fait boire du lait au bébé; Le kangourou fait manger du miel au petit ours.

c) causatifs lexicaux-tuer $=$ faire mourir et montrer $^{21}=$ faire voir. Pour ce qui est de ces deux verbes, notre objectif est d'explorer l'apparition des erreurs par surgénéralisation

18. Sur le plan syntaxique, ces verbes se caractérisent par une valence stable et ils désignent une action qui part du sujet (plan sémantique). Pour ces raisons, il est impossible de les utiliser sans un verbe causatif (ex. The teacher *played the children.).

19. Nous n'avons trouvé aucun emploi transitif (causatif + ) de ce verbe en français; tandis que l'anglais se montre plus souple à son égard autorisant la suppression de l'auxiliaire causatif make sous certaines conditions (ex. *Dance me to the end of love).

20. Sur le plan syntaxique, ce verbe se caractérise par une valence instable et il exprime une action qui vise ou atteint le sujet (plan sémantique). Ses emplois transitifs (causatif + ) en français sont attestés au Moyen Âge et au XVI ie siècle (Gougenheim, 1929: 324).

21. Gaston Paris s'aperçoit d'un fait curieux en ancien français, notamment l'emploi de faire + Vinf avec la valeur d'un verbe simple lorsque ce dernier équivaut une locution formée de faire et d'un infinitif. Par exemple, faire mener $=$ mener $($ mener $=$ faire aller $)$; faire montrer $=$ montrer (montrer $=$ faire voir). Ce phénomène aurait son origine dans une sorte de superposition inconsciente de deux tours équivalents (cf. Gougenheim, 1929: 333) (ex. «[...] et me proient que je lour face moustrer le saint roi, dem). 
(ex. : Ulysse, il *fait montrer le village à Hercule.). Cellesci marquent une étape importante dans le développement langagier des enfants. En faisant appel à leurs capacités cognitives générales, ainsi qu'à leurs habiletés sociocognitives, les jeunes locuteurs se transforment en créateurs de nouvelles constructions (en syntaxe, ce phénomène se fait sentir à partir de 3 ans jusqu'à l'âge de 4-6 ans) (Tomasello, 2003).

Les facteurs complexité et fréquence jouent un rôle crucial dans le processus d'acquisition d'un fait de langue (Tomasello, 2003). Le tableau 1 ci-dessous illustre ces deux critères :

Classes de verbes À Tester

Intransitifs

Transitifs

Causatifs

lexicaux

$$
\begin{aligned}
& \text { avec } \\
& \text { faire } \\
& \text { sans } \\
& \text { faire } \\
& \text { boire } \\
& \text { manger } \\
& \text { tuer } \\
& \text { montrer }
\end{aligned}
$$$$
\text { rire }
$$$$
\text { pleurer }
$$$$
\text { tomber }
$$$$
\text { danser }
$$$$
\text { + FRÉQUENTS }
$$$$
\text { - FRÉQUENTS }
$$$$
918
$$$$
711
$$$$
243
$$$$
26273
$$

Tableau 1 - Classes de verbes inclus dans le protocole expérimental selon leurs propriétés syntaxiques et leur fréquence

Pour mener l'expérimentation, nous avons conçu une tâche de production, une de compréhension et une d'imitation (McDaniel, McKee \& Smith Cairns, 1998).

La tâche de production est présentée sous forme de brefs clips vidéo (des extraits de dessins animés), que les enfants observent sur l'ordinateur. Ces clips montrent des situations potentiellement causatives où un personnage fait agir un autre. L'objectif de la tâche est de voir si, ou bien, à quel point les jeunes locuteurs entre 4 et 6 ans sont capables de produire correctement le PC faire + Vinf. Pour éliciter le factitif, nous posons trois questions principales toujours dans le même ordre: 1/sur le causateur dans la situation causative (ex.: Qu'est-ce qu'elle fait, la grenouille? $\rightarrow$ Elle gonfle ses 
joues.); 2/sur le causataire de la situation causative (ex.: Qu'est-ce qu'il fait, Tarzan? $\rightarrow$ Il pleure.); 3/sur ce que le causateur fait au causataire (ex. : Qu'est-ce que la grenouille fait à Tarzan? $\rightarrow$ Elle le fait pleurer.). D'une part, cet ordre d'apparition des questions permet de sensibiliser l'enfant en attirant son attention, d'abord, sur les actions non causatives (questions 1 et 2), puis sur la situation causative (question 3). D'autre part, il nous permet de faire des hypothèses sur les étapes d'acquisition par l'enfant du PC faire + Vinf. En d'autres termes, si le factitif est produit spontanément, avant toutes nos questions, ou bien en réponse à la première question, alors il n'y aura pas de doute sur la disponibilité du PC faire + Vinf en production. Par contre, si le factitif apparait suite à la troisième question, on pourrait avancer l'hypothèse que l'enfant a toujours besoin d'aide extérieure (d'indices) pour produire correctement cette construction complexe.

La tâche de compréhension se déroule sous forme de jeux d'interaction entre l'enquêtrice et l'enfant. Elle a pour objectif d'explorer la compréhension du factitif chez les jeunes locuteurs. À cet effet, l'enquêtrice produit un énoncé (à la forme impérative vs interrogative) évoquant une situation causative, et l'enfant est amené à jouer la scène à l'aide de figurines (ex.: Voilà un bébé et une maman. La maman fait boire le bébé. A toi maintenant, fais comme la maman! vs Est-ce que tu peux faire comme la maman?).

La tâche d'imitation avec changement du lexique se déroule également sous forme de jeux d'interaction entre l'enquêtrice et l'enfant. Son objectif est l'exploration de l'imitation productive chez les jeunes apprenants. L'enquêtrice réalise une action causative à l'aide de figurines et, en même temps, elle produit le modèle de formation des factitifs. Puis elle répète la même action avec d'autres figurines. L'enfant est amené à reproduire le modèle adulte en impliquant de nouveaux items lexicaux. Cette tâche expérimentale pourrait être illustrée par le schéma suivant: $\underline{\mathbf{A}}$ factitif $\underline{\mathbf{B}} \rightarrow \underline{\mathbf{C}}$ même factitif $\underline{\mathbf{D}}$ (ex.: Regarde bien, je vais te montrer quelque chose: La maman fait boire le bébé. Et maintenant, dis-moi, qu'est-ce qu'il fait, le papa? (l'enquêtrice montre à l'enfant le papa qui fait boire la grande sœur). $\rightarrow$ Le papa fait boire la grande sceur.). 


\section{Premiers résultats de l'expérimentation}

Dans cette section, nous présenterons les résultats constatés au stade actuel de notre recherche sur le prédicat complexe faire + Vinf dans le langage des enfants unilingues francophones entre 4 et 6 ans. D'abord, nous rendrons compte du pourcentage de réussite des tâches de production et d'imitation. Ensuite, nous ferons un bref bilan sur les compétences des enfants en compréhension. Enfin, nous comparerons nos résultats avec ceux de l'étude de Sarkar (2002) et sur cette base nous tenterons de voir dans quelle mesure les étapes de l'acquisition du prédicat complexe faire + Vinf pourraient reproduire celles de sa grammaticalisation.

Les résultats que nous avons obtenus à l'issue des tâches de production et d'imitation avec changement du lexique sont très variés. Au cours de ces deux épreuves, chacun des 18 participants ( 9 enfants par tranche d'âge) est amené à produire et puis à imiter six constructions factitives (faire rire, faire pleurer, faire tomber, faire danser, faire boire, faire manger) et deux causatifs lexicaux (tuer, montrer). Le tableau 2 ci-dessous résume les catégories de réponses fournies par les enfants des deux tranches d'âge $\left(\mathrm{N}_{1}=4-5\right.$ ans et $\mathrm{N}_{2}=5-6$ ans):

\begin{tabular}{|c|c|c|c|c|}
\hline \multirow[t]{2}{*}{ CATEGORIES DE RÉPONSES } & \multicolumn{2}{|c|}{$\begin{array}{l}N_{1} \text { (ENTRE } 4 \text { ET } \\
5 \text { ANS) }\end{array}$} & \multicolumn{2}{|c|}{$\begin{array}{l}\mathrm{N}_{2} \text { (ENTRE } 5 \\
\text { ET } 6 \text { ANS) }\end{array}$} \\
\hline & PROD. & IMIT. & PROD. & IMIT. \\
\hline $\begin{array}{l}\text { faire }+ \text { Vinf }+2 \text { arg. justes: } \\
\text { Le papa fait rire la fille }\end{array}$ & 37,5 & 40,7 & 7,4 & 29,6 \\
\hline $\begin{array}{l}\text { faire }+ \text { Vinf }+2 \text { arg. }+ \text { hésitation } \\
\text { sur le genre: } \\
* \text { Elle *le fait rire }\end{array}$ & 2 & 13 & 0 & 7,4 \\
\hline $\begin{array}{l}\text { faire }+ \text { Vinf }+2 \text { arg. dont causataire } \\
\text { erroné au datif: } \\
\text { ll } * \text { lui fait rire }\end{array}$ & 2 & 5,6 & 14,9 & 3,7 \\
\hline $\begin{array}{l}\text { faire }+ \text { Vinf }+1 \text { arg. juste } \\
+1 \text { arg. absent: }\end{array}$ & 2 & 2 & 3,7 & 0 \\
\hline $\begin{array}{l}\text { Le papa fait rire }+\emptyset \\
\text { ellipses et transitivations: } \\
\text { pleurer le bébé; pleurer; il * rit la fil }\end{array}$ & 0 & 9,3 & 1,9 & 14,9 \\
\hline donner à manger/à boire & 13 & 7,4 & 14,9 & 11 \\
\hline surgénéralisations: & 6 & 0 & 0 & 6 \\
\hline
\end{tabular}




$\begin{array}{lcccc}n l * \text { fait montrer le village à Hercule } & & & & \\ \begin{array}{l}\text { réponses partielles ou absence } \\ \text { de réponse }\end{array} & 37,5 & 22 & 57,2 & 27,4 \\ & 100 & 100 & 100 & 100\end{array}$

Tableau 2 - Pourcentage de réussite des tâches de productio et d'imitation avec les cibles faire + Vinf et causatifs lexicaux

Le tableau 2 ci-dessus montre qu'à ce stade du développement langagier (entre 4 et 6 ans) la construction factitive ne peut pas être considérée comme entièrement maitrisée. En production, on constate un écart considérable entre les deux classes d'âge concernant les emplois conventionnels (avec les deux arguments causateur et causataire présents et justes) de la cible faire + Vinf. Celui-ci s'élève à $37,5 \%$ chez les enfants âgés de 4 à 5 ans, tandis que chez les enfants entre 5 et 6 ans il atteint à peine $7,4 \%$ (ligne 1 , tableau 2 ). La différence entre les résultats fournis par les enfants des deux tranches d'âge se conserve en imitation (40,7\% chez les petits vs $29,6 \% \mathrm{chez}$ les plus âgés).

Le changement des items lexicaux a un impact considérable sur l'hésitation au niveau du genre des arguments. Le degré d'apparition de ces erreurs augmente de $2 \%$ (en production) à $13 \%$ (en imitation), chez les enfants entre 4 et 5 ans et de 0 (en production) à $7,4 \%$ (en imitation), chez les enfants âgés de 5 à 6 ans (ligne 2 du tableau).

Une autre différence importante entre les productions des sujets interviewés est observable au niveau syntaxique ( $2 \%$ chez les petits vs 14,9\% chez les plus âgés) (ligne 3, tableau 2); les enfants entre 5 et 6 ans ont tendance à récupérer le causataire comme datif lorsque le verbe de départ est monovalent (à 1 actant) (ex. : Il rit.-monovalent $\rightarrow$ Il fait rire la fille. $\rightarrow$ Il la fait rire.- bivalent, ce qui sera produit comme Il *lui fait rire.). Au cours de la tâche d'imitation, l'écart entre les résultats fournis par les enfants des deux tranches d'âge diminue $(5,6 \%$ chez les petits $v s 3,7 \%$ chez les plus âgés).

Dans le corpus recueilli, nous avons relevé des emplois sporadiques de la construction factitive avec un argument juste (le causateur) et un argument absent (le causataire) (ligne 4 , tableau 2). Le nombre de ces occurrences non 
conventionnelles s'élève à $2 \%$ en production comme en imitation chez les enfants âgés de 4 à 5 ans. Apparemment, à ce stade du développement langagier les jeunes locuteurs sont capables aussi bien de produire que de supprimer l'un des arguments du prédicat complexe faire + Vinf; la construction factitive ne serait donc pas encore appropriée dans son intégralité. En revanche, les énoncés du type Le papa fait rire $+\varnothing$ sont plus fréquents en production chez les participants âgés de 5 à 6 ans $(3,7 \%)$; ces emplois déviants disparaissent complètement en imitation. Il en résulte qu'entre 5 et 6 ans, les enfants se montrent attentifs à exprimer l'instigateur du procès et l'action elle-même plutôt que l'agent.

Lors de la tâche d'imitation avec changement du lexique, un fait curieux se remarque tout de suite; il s'agit de la hausse du nombre des énoncés elliptiques (tomber la fille, tomber) et des occurrences de transitivation causative (ex.: Il * rit la fille.). Celui-ci augmente de $1,9 \%$ (en production) à $14,9 \%$, chez les enfants âgés de 5 à 6 ans et de 0 (en production) à 9,3 $\%$, chez les plus jeunes (ligne 5 du tableau). Ce phénomène pourrait être dû à des raisons communicatives, c'est-à-dire que l'enfant fait exprès d'éviter la répétition du verbe factitif faire dans ses énoncés, puisque celui-ci est déjà présent dans la question de l'enquêtrice.

Nous avons également relevé l'emploi des constructions bi-clausales (moins grammaticalisées) donner à manger/à boire (ligne 6, tableau 2); celles-ci apparaissent avec presque la même fréquence chez les participants des deux tranches d'âge (13\% chez les enfants entre 4 et 5 ans $v s \quad 14,9 \%$ chez les enfants entre 5 et 6 ans). Tous les sujets interviewés se montrent capables de bien manier ces structures complexes à deux (voire à trois) arguments (ex. Il donne à manger $\underline{\text { du miel }}$ au petit ours.). L'explication, que nous pourrions en donner consiste dans la nature bi-clausale des deux constructions; chacun des verbes composants est suivi de son propre argument. En revanche, la fusion des prédicats implique une restructuration, une réanalyse des arguments (ce n'est que le verbe auxiliaire qui s'attache les clitiques), ce qui retarderait le processus d'acquisition (cf. supra). 
La tâche d'imitation se caractérise par une baisse générale du nombre des constructions moins grammaticalisées donner à manger/à boire (de $13 \%$ en production à $7,4 \%$, chez les enfants entre 4 et 5 ans et de $14,9 \%$ en production à $11 \%$, chez les enfants âgés de 5 à 6 ans). Ce fait n'a rien de surprenant, puisque les enfants sont amenés à produire faire + Vinf après avoir entendu le modèle adulte fourni par l'enquêtrice.

Ce sont les enfants âgés de 4 à 5 ans qui ont tendance à commettre des erreurs par surgénéralisation en production $(6$ $\%$ ) (ligne 7, tableau 2). Ils appliquent le schéma faire + , sans tenir compte du fait que dans cette construction, l'espace vide réservé au verbe à l'infinitif ne peut pas être occupé par une variante lexicale du prédicat complexe faire $+\operatorname{Vinf}$ (montrer $=$ faire voir). Les erreurs par surgénéralisation disparaissent complètement lors de la tâche d'imitation. En revanche, les enfants âgés de 5 à 6 ans ne fournissent aucun exemple de surgénéralisation en production, tandis qu'en imitation le nombre de ces emplois déviants est estimé à $6 \%$. Apparemment, les apprenants avancés sont conscients de ce que nous leur demandons de faire au cours de l'expérimentation centrée sur faire + Vinf et sous cette influence ils font émerger des erreurs par surgénéralisation sans tenir compte de la présence du modèle adulte.

Un dernier point sur lequel les énoncés des participants fournis lors de la tâche de production se différencient concerne le nombre des réponses partielles (portant sur l'action du causateur qui fait agir le causataire), ainsi que les cas d'absence de réponse cohérente (qui ne prend en compte aucun des éléments de la situation causative). Ces erreurs sont estimées à $57,2 \%$ chez les enfants plus âgés, tandis que chez les plus jeunes elles atteignent 37,5\% (ligne 8 du tableau). Apparemment, l'émergence de la construction factitive est difficile et, de plus, la situation causative n'est pas toujours conceptualisée. Cette dernière $a$ un caractère particulier: elle ne présente pas un agent exécutant une action ordinaire par lui-même (ex. Jean rit; Jean court.). Elle évoque plutôt l'instigateur, celui qui fait quelque chose pour qu'un autre actant agisse. Il y aurait donc une action intermédiaire (cognitivement présente, mais qui n'est pas exprimée linguistiquement) 
(ex. Jean fait rire Marie: 1/action I (sous-entendue) = Jean fait une grimace; 2/action $2=$ Marie rit. $3 /$ Jean fait rire Marie). D'un point de vue cognitif, la présence de cette étape intermédiaire rend la situation causative difficile à conceptualiser, ce qui retarderait l'acquisition de faire + Vinf. Dans le même sens vont les réflexions de Bratanova (2006), à savoir que tout ce qui est cognitivement complexe, dans la langue, est exprimé par une structure complexe (principe de l'iconicité).

$\mathrm{Au}$ cours de la tâche d'imitation, le nombre des réponses partielles (ou absentes), fournies par les enfants des deux tranches d'âge diminue (22\% chez les petits vs $27,4 \%$ chez les plus âgés).

Finalement, les résultats illustrés dans le tableau 2 peuvent paraître paradoxaux; ils témoignent d'une meilleure maîtrise générale de la construction factitive faire $+\operatorname{Vinf}$ chez les enfants âgés de 4 à 5 ans. Ce phénomène pourrait être expliqué par la tendance à la créativité, à la diversification des moyens d'expression chez les enfants entre 5 et 6 ans. En revanche, les plus jeunes locuteurs, qui maîtrisent moins les outils de la langue chercheraient plutôt à reproduire le modèle adulte.

Comme indiqué dans l'introduction de la présente section, nous nous intéressons également aux compétences des enfants en compréhension. Le tableau 3 ci-dessous illustre les résultats obtenus avec les deux cibles faire + Vinf et causatifs lexicaux:

Catégories de comportements $\mathrm{N}_{1}$ (Entre 4 et $\mathrm{N}_{2}$ (Entre 5 et

$$
5 \text { ANS) } 6 \text { ANS) }
$$

\begin{tabular}{|c|c|c|c|c|}
\hline & $\begin{array}{l}\text { FAIRE } \\
+ \text { VINF }\end{array}$ & $\begin{array}{l}\text { CAUS. } \\
\text { LEX. }\end{array}$ & $\begin{array}{l}\text { FAIRE } \\
+ \text { VINF }\end{array}$ & $\begin{array}{l}\text { CAUS } \\
\text { LEX. }\end{array}$ \\
\hline 2 (compréhension totale) & 64,8 & 94,4 & 59,3 & 77,8 \\
\hline 1 (signe de compréhension) & 5,6 & 0 & 14,8 & 5,6 \\
\hline 0 (absence de tout signe & 29,6 & 5,6 & 25,9 & 16,6 \\
\hline de compréhension) & 100 & 100 & 100 & 100 \\
\hline
\end{tabular}

Tableau 3 - Pourcentage de réussite de la tâche de compréhension avec les cibles faire + Vinf et causatifs lexicaux

D'après le tableau 3 ci-dessus, ce sont encore les enfants entre 
4 et 5 ans qui fournissent les meilleurs résultats en compréhension avec la cible faire + Vinf. $(64,8 \%$ vs $59,3 \%$ chez les plus âgés) (ligne 1 du tableau). Les participants âgés de 5 à 6 ans préfèrent donner des signes de compréhension sans exécuter l'action causative (ex. Elle l'a tapé, la maman, en réponse à La maman fait pleurer le bébé. A toi maintenant, fais comme la maman!) (14,8\% vs $5,6 \%$ chez les petits) (ligne 2, tableau 3). Ce phénomène est à lier, à notre avis, au fait qu'entre 5 et 6 ans les enfants commencent à sortir de la période où les jeux sont centrés sur le maniement de différentes figurines (une tendance encore plus évidente chez les garçons).

Les participants âgés de 4 à 5 ans se montrent meilleurs en compréhension même avec la cible causatifs lexicaux $(94,4 \%$ vs $77,8 \%$ chez les plus âgés) (ligne 1 , tableau 3 ). Les enfants entre 5 et 6 ans gardent leur préférence aux explications des actions causatives plutôt qu'à leur exécution $(5,6 \%)$ (ligne 2 du tableau).

Finalement, nous pourrions dire qu'à ce stade du développement langagier, la construction factitive est disponible en compréhension. Celle-ci est cognitivement présente, puisque les enfants âgés de 4 à 6 ans se montrent capables de la reconnaitre dans l'environnement langagier (l'input).

Les démarches méthodologiques, que nous avons adoptées pour mener notre expérimentation, ainsi que nos premiers résultats vont au-delà de ceux rapportés par Sarkar (2002). Elle explore la capacité des jeunes locuteurs à comprendre et à produire la construction factitive (spontanément, ou bien après avoir entendu le modèle adulte). L'analyse quantitative qu'elle applique lui permet d'esquisser les trois principales étapes dans l'acquisition du PC faire $+\operatorname{Vinf}\left(\right.$ (p. 191) ${ }^{22}$ :

a) le verbe factitif faire est souvent omis (ex. Je danse le petit chat.). L'auteur précise qu'entre la période de suppression

22. L'analyse quantitative concerne les données recueillies auprès de quatre des huit enfants monolingues francophones participant dans l'expérimentation de Sarkar (2002). Nous ne disposons pas de l'intégralité de ces résultats au moment de la rédaction du présent article (pour plus de détails sur la question voir Sarkar, 2000). 
massive de faire (avant 2 ans) et celle de son apparition consécutive, il y aurait une étape intermédiaire au cours de laquelle certains enfants sont plutôt disposés à le supprimer, tandis que d'autres sont plutôt disposés à le produire (mostly omitting or mostly supplying) (p. 180).

b) clitiques mal placés, alors faire + Vinf n'est pas encore considéré comme $\mathrm{PC}$, mais comme une périphrase (ex. ${ }^{*} J e$ fais les sauter.). Sarkar précise que ce type d'erreurs est rare dans son corpus. De plus, le mauvais placement des clitiques est toujours accompagné par de nombreux exemples d'emplois conventionnels.

c) construction stabilisée (ex. Puis, je vais le faire sauter sur le bébé.).

Notre corpus contient très peu d'occurrences confirmant les deux premières étapes-omission relative de faire et interprétation bi-clausale (à deux prédicats) de la construction faire + Vinf. Ce fait serait dû à l'âge plus avancé de nos sujets interviewés (entre 4 et 6 ans). En revanche, les données que nous avons recueillies et traitées à l'heure actuelle de notre recherche vont à l'encontre de l'affirmation de Sarkar (2002), selon laquelle l'émergence du prédicat complexe faire + Vinf se fait entre 2 et 4 ans; la maîtrise de la construction factitive atteint $50 \%$ chez les enfants âgés de 4 à 5 ans et à peine 32,4 $\%$ chez les enfants entre 5 et 6 ans (y compris les occurrences avec hésitation au niveau du genre du causateur et du causataire, ainsi que celles avec placement syntaxiquement erroné du causataire).

Pour l'instant, nous ne disposons pas encore de données concernant l'acquisition des constructions causatives en bulgare: $1 /$ «karam njakogo da conjonction + pee $\left(V_{\text {presen }}\right) »=$ inciter qn. à ce qu'il chante (= faire chanter qn.);2/«davam na njakogo da jade» = donner à manger $\grave{a} q n .=(=$ faire manger qn.), «davam na njakogo da pie» = donner à boire à qn. (=faire boire qn.). Ces périphrases sont moins grammaticalisées qu'en français, ce qui nous fait supposer que les enfants bulgares auraient moins de difficultés à les produire au même âge (entre 4 et 6 ans). Tous ces questionnements vont faire l'objet de notre étude contrastive sur l'acquisition du PC faire + Vinf en français et de ses équivalents causatifs en bulgare. 
Les premiers résultats de notre étude sur l'acquisition du prédicat complexe faire $+\operatorname{Vinf}$ nous permettent de tirer quelques conclusions intéressantes.

Premièrement, nous ne disposons d'aucun indice de monoclausalité précoce au niveau de la construction factitive, ce qui n'a rien de surprenant vu l'âge de nos sujets interviewés (de 4 à 6 ans). Il en est de même pour l'interprétation bi-clausale de faire + Vinf. Les seules apparitions de structures complexes moins grammaticalisées (à deux prédicats) dans le langage enfantin concernent donner à manger/à boire, mais ces constructions sont également bi-clausales chez les adultes.

Deuxièmement, nos données, ainsi que celles recueillies par Sarkar (2002) n'apportent pas de preuves convaincantes concernant la fluctuation entre interprétations mono- et biclausale de faire + Vinf.

Troisièmement, les résultats dont nous disposons nous laissent supposer qu'à ce stade du développement langagier (entre 4 et 6 ans) la construction factitive faire + Vinf en français n'est pas encore acquise. Elle est sans aucun doute cognitivement présente et, par conséquent, facile à reconnaitre dans l'environnement langagier (l'input). Toutefois, les enfants éprouvent encore des difficultés à la retrouver par eux-mêmes et ce, voire en présence du modèle adulte. Dans certains cas, ils parviennent à la produire correctement, dans d'autres, ils cherchent à la remplacer, ou bien, ils en font tomber un constituant (causateur, causataire, verbe factitif faire).

Les résultats que nous avons obtenus témoignent d'une meilleure maîtrise générale (en production, compréhension et imitation) chez les enfants entre 4 et 5 ans. Au stade actuel de notre recherche, nous ne pouvons pas donner une explication définitive à ce phénomène paradoxal.

Finalement, notre hypothèse de départ d'après laquelle l'évolution du prédicat complexe faire $+V i n f$ dans le langage enfantin pourrait reproduire certaines étapes de sa grammaticalisation ne se confirme pas pour 1'instant. Il serait intéressant de mener des études plus approfondies sur la question auprès d' enfants d'âges variés. 


\section{RÉfÉRENCES}

ABEILlÉ, A. \& GodARD, D. (2003): Les prédicats complexes. Les langues romanes. Problèmes de la phrase simple. Sous la dir. de D. Godard, Paris, CNRS (éd).

AlsinA, A. (1996): The Role of Argument Structure in Grammar. Evidence from Romance, Stanford, CA, CSLI Publications.

Bratanova, B. (2006) : Between Cognition and Cultural Discourse: A Liminal Approach to Causation. Language. $<$ www.inst.at/trans/16Nr/05_6/bratanova16.htm $>$.

CHANSON DE ROLAND (LA), Paris, GF-Flammarion.

CICÉRon (I ${ }^{\text {er }}$ siècle av. J.-C.): Epistulae ad Atticum, t. 2, Livres VII-XI, Paris, Garnier (1938).

COMBETTES, B. et al. (2003): Introduction: grammaticalisation et changement linguistique. Verbum, XXV, 2003, 3, Nancy, Presses universitaires de Nancy, 225-240.

COMRIE, B. (1976): The syntax of causative constructions: cross-language similarities and divergences. Shibatani, $M$. (éd.), Syntax and Semantics, Volume 6: The Grammar of Causative Constructions, New York, Academic Press, 261-312.

Creissels, D. (2006): Syntaxe générale: une introduction typologique, t. 1, Paris, Lavoisier.

Dixon, R.M.W. (2000): A Typology of Causatives: from Syntax and Meaning. R.M.W. Dixon \& A. Aikhenvald (Ed.). Changing Valency. Case Studies in Transitivity: 3088. Cambridge University Press.

GAATONE, D. (1976): Les pronoms conjoints dans la construction factitive. Revue de linguistique romane, $n^{\circ}$ 159-160, Strasbourg, G. Straka, Palais de l'Université, 165-182.

GAWEłKo, M. (2006): Remarques sur les constructions causatives du type «faire + inf.» dans les langues romanes et quelques autres. Acta Linguistica Hungarica, vol. 53 (2), Springer, Kluwer Academic Plublishers, 117-138.

GougENHEIM, G. (1929): Étude sur les périphrases verbales de la langue française, Paris, Les Belles Lettres.

Heine, B. (1993): Auxiliaries. Oxford, Oxford University Press. 
HOPPER, P. (1987): Emergent grammar. Berkeley Linguistics Society, 13, Berkeley, University of California, 139-157.

ILIESCU, M. (1996): Le factitif dans les langues romanes. Romanistik in Geschichte und Gegenwart, 2,1, Hamburg, Helmut Buske Verlag, 3-13.

KURYLOWICZ, J. (1965): The evolution of grammatical categories. Esquisses linguistiques, 1975, München, Fink, 3854.

LAMIROY, B. (1999) : Auxiliaires, langues romanes et grammaticalisation. Langages, 135, Paris, Larousse, 33-45.

LEVIN, B. \& RAPPAPORT HOVAV, M. (1995): Unaccusativity. At the Syntax-Lexical Semantics Interface, Cambridge (Mass.); London, MIT Press.

LuCRÈCE CARUS ( ${ }^{\text {er }}$ s. av. J.-C.) : De rerum natura, t. 1, Livres I-III, Paris, Les Belles Lettres (1968).

McDaniel, D., McKee, C. \& Smith Cairns, H. (1998): Methods for Assessing Children's Syntax, Cambridge (Mass.); London, MIT Press.

MEILleT, A. (1912-1982): L'évolution des formes grammaticales. Linguistique historique et linguistique générale. Paris, Champion, 1948, 130-148.

Nilsson-Ehle, H. (1948): Observations sur la soudure syntaxique du groupe Faire + Infinitif. Studia linguistica, Année II, Oxford, Blackwell, 93-118.

Novakova, I. (2002): Le factitif français: approche syntaxique, sémantique et contrastive (français-bulgare). Travaux neuchâtelois de linguistique (TRANEL), 2002, 37, Neuchâtel, Institut de linguistique, 93-113.

Novakova, I. (2006): Causative Mechanisms in Bulgarian and French. Syntax of World's Languages, 14-17 sept. 2006, Lancaster University, UK.

Perlmutter, D. (1978): Impersonal Passives and the Unaccusative Hypothesis. Proceedings of the 4th annual meeting of the Berkeley Linguistic Society, BLS, Berkeley, (CA.), 157-189.

PeYraube, A. (2002): L'évolution des structures grammaticales. Langages, 146, Paris, Larousse, 46-58.

Plaute (III ${ }^{\mathrm{e}}-\mathrm{II}^{\mathrm{e}} \mathrm{s}$. av. J.-C.) : Comédies (Epidicus), t. 3, Paris, Les Belles Lettres (1935). 
SARKAR, M. (2000): Saute ça / "Jump this !": The acquisition of the faire faire causative by first and second language learners of French. Unpublished doctoral dissertation, Montreal, Concordia University.

SARKAR, M. (2002): Saute ça/Jump this !: The acquisition of the faire faire causative by first and second language learners of French. Annual Review of Language Acquisition, vol. 2, Amsterdam, John Benjamins Publishing Company, 157-201.

Simone, R. \& Cerbasi, D. (2001): Types and diachronic evolution of romance causative constructions. Romanische Forschungen, 113/3, Frankfurt am Main, Klostermann, V., 441-473.

TESNiĖRE, L. (1969): Éléments de syntaxe structurale, Paris, Klincksieck (éd.).

TOMASEllo, M. (2003): Constructing a language: a usagebased theory of language acquisition, Cambridge (Mass.); London, Harvard University Press.

VIRGILE (I ${ }^{\text {er }}$ siècle av. J.-C.): Enéide, t. 1, livres I-IV, Paris, Les Belles Lettres (1992). 